This is a manuscript, which has been accepted for publication.

Published in: Proceedings of the Institution of Mechanical Engineers, Part F: Journal of Rail and Rapid Transit. October 2020.

\title{
Application and Improvement of a Direct Method Optimization Approach for Battery Electric Railway Vehicle Operation
}

\section{Authors and Affiliations}

Moritz Schenker ${ }^{1}$, Toni Schirmer ${ }^{1}$, Holger Dittus ${ }^{1}$

${ }^{1}$ German Aerospace Center (DLR), Stuttgart, Germany

\section{Corresponding Author's Contact Detail}

Moritz Schenker, German Aerospace Center (DLR), Institute of Vehicle Concepts,

Pfaffenwaldring 38-40, 70569 Stuttgart, Germany

moritz.schenker@dlr.de

+4971168628410

\section{Acknowledgements}

The authors would like to thank Athanasios Iraklis, who initially started the implementation of a direct method optimization algorithm during his time at DLR.

\section{Declaration of Conflicting Interests}

The Authors declare that there is no conflict of interest.

\section{Funding}

This research was conducted within the DLR Next Generation Train project. 
This is a manuscript, which has been accepted for publication.

Published in: Proceedings of the Institution of Mechanical Engineers, Part F: Journal of Rail and Rapid Transit. October 2020.

Publisher Version:10.1177/0954409720970002

\begin{abstract}
While the largely electrified rail network allows for direct utilization of renewable energy sources, there is still a considerable share of diesel-powered trains operating on non- and partly electrified tracks. To replace these, the more sustainable alternatives such as battery electric railway vehicles need to present a viable option with sufficient range. This paper aims to adapt and improve an existing optimization algorithm, previously used with diesel-powered trains, for the operation of battery electric railway vehicles. In this new approach, battery control is optimized alongside train control, utilizing a direct method solver to find the minimum energy trajectory. Furthermore, a detailed train model is implemented that is designed for operation on partly electrified tracks. To yield a highly accurate, yet also sufficiently fast algorithm, a numerical analysis is conducted and the parameters of the algorithm are determined accordingly. Finally, the application of the adapted algorithm on a use case in Germany shows that both velocity profile and control adapt in a way that minimizes utilization of the battery. The results indicate that the proposed algorithm presents a reliable and robust method to obtain minimum energy controls for battery electric railway vehicles with any electrification pattern.
\end{abstract}

\title{
Keywords
}

Direct Method, Optimal Control, Battery Electric Railway Vehicles, Battery Control, Partly Electrified Tracks, Railway Optimization, Minimum Energy Trajectories

\section{Introduction}

In the context of the international fight against climate change sustainable means of transport and locally emission-free propulsion systems gain more importance, on road as well as on rail. One emerging technology that is currently in development within rail industry is the Battery Electric Multiple Unit (BEMU). ${ }^{1}$ BEMUs utilize a hybrid approach with overhead line equipment and an onboard Energy Storage System (ESS) in form of a battery. ${ }^{2}$

Since the energy-saving potential due to optimized operation is substantial in battery powered railway applications, ${ }^{3}$ there is a need to extend optimization methods to the new use cases. While there is extensive literature on optimization with onboard ESS on either completely electrified routes or routes without any electrification, ${ }^{4-11}$ only one work is known to us that considers partly electrified tracks (cf. Xiao et al. ${ }^{12}$ ). However, this pseudo-spectral optimization method was focused on a hybrid ESS for tram operation. For a more general applicability an algorithm is needed that is easy to modify and able to deal with complex problem set. For this aim, Macian et al. introduced an algorithm for the optimal control of a diesel-powered train, which showed improved performance compared to well-established methods. ${ }^{13}$

The work presented in this paper should answer the following question: Is the gradient-based Direct Method (DM) optimization approach introduced by Macian et al. in 2018 applicable on scenarios with battery electric railway vehicles and partly electrified tracks? At the same time, this paper attempts to obtain more realistic results by detailed modeling of the train, including auxiliary powers and load-dependent efficiency curves, and respect ride comfort restrictions 
This is a manuscript, which has been accepted for publication.

Published in: Proceedings of the Institution of Mechanical Engineers, Part F: Journal of Rail and Rapid Transit. October 2020.

Publisher Version:10.1177/0954409720970002

such as ac- and deceleration limits as mentioned in the outlook by Macian et al. ${ }^{13}$ Additionally, a numerical study and parameter calibration was conducted to reduce the computational effort and gain a fast algorithm.

After a short introduction to the previous work of Macian et al., our model extensions and adaptions are presented. Afterwards, the parameter optimization is shown, followed by the presentation of a use case on a real route in Germany.

\section{Background - Direct Method}

The development of a new optimization algorithm for railway operation by Macian et al. was driven by three main goals: ${ }^{13}$

1. To obtain optimal operation for a train model with any complexity,

2. The possibility to include any kind of constraint in a straightforward manner and

3. To keep computational requirements as low as possible.

To achieve this, they applied three different methods on the Optimal Control Problem (OCP) of a diesel-powered train and conducted a benchmark. Pontryagin's Minimum Principle (PMP) and Dynamic Programming (DP), both widely used in railway trajectory optimization, failed at least one of the goals mentioned above. DP resulted in very high computational requirements due to curse of dimensionality and violated equality constraints. PMP, on the other hand, failed to yield results for the OCP because the calculation of costates (Lagrangian multipliers) gets too complex. This could only be solved by a simplification via pre-fixed operation modes and, therefore, conflicts with the first two goals.

The DM approach of constructing a large and sparse Non-Linear Programming (NLP) problem via collocation by Macian et al., however, fulfilled all goals. With this method, the continuous spatial domain of the whole route is discretized into a numerical grid and the optimal trajectory is approximated by known functions within steps between collocation/grid points (e.g. polynomials or piecewise functions). With a system model (differential equations and constraints) eligible function values can be determined. The optimal values are then calculated by an NLP solver.

The OCP can be formulated with the following mathematical description:

$$
\begin{gathered}
\min _{x} f(x), \text { s.t. } \\
c_{1} \leq c(x) \leq c_{\mathrm{u}} \\
x_{\mathrm{l}} \leq x \leq x_{\mathrm{u}}
\end{gathered}
$$

Here, $f(x)$ denotes the objective function, which is to be minimized. In Macian's case this is the integral of fuel consumption rate along the route. ${ }^{13}$ The solution vector $x$ consists of all states and actuators at the grid points, in this case velocities and power control settings (for both traction and braking). The behavior of the solution variables is defined by the differential equation (DE) of motion and boundary conditions such as zero velocity at stops. It is furthermore bound by lower and upper limits $\left(x_{1}\right.$ and $x_{\mathrm{u}}$ : e.g. speed limits and physical system bounds). Macian et al. considered additional system constraints $c(x)$ in form of power and force limits (path constraints given in each grid point) as well as a timetable (integral constraint 
This is a manuscript, which has been accepted for publication.

Published in: Proceedings of the Institution of Mechanical Engineers, Part F: Journal of Rail and Rapid Transit. October 2020.

Publisher Version:10.1177/0954409720970002

between two stops). ${ }^{13}$ The NLP formulation allows for all DEs and constraints to be transcribed into one vector. Since the utilized NLP solver Interior Point OPTimizer (IPOPT) ${ }^{14}$ is gradientbased, first (gradient of objective function and Jacobian of constraints) and second (Hessian of both, not used by Macian) derivatives need to be provided or approximated. With the additional knowledge of derivatives, the optimization speed can be increased considerably. Therefore, objective function and constraints have to be twice continuously differentiable. Macian et al. achieved this by fitting the input functions with Fourier series and Gaussian functions. ${ }^{13}$

Macian et al. achieved to handle the large problem with a straightforward implementation of the constraints. Utilizing a state-of-the-art open access NLP-solver, the optimization converged fast and with low computational requirements in a robust process. Additionally this method allows using previous solutions for increased convergence speed. Minor drawbacks are that NLP solvers only yield local instead of global optima and results and convergence behavior depend on the initial solution. The restriction of twice continuously differentiable input functions bounds the applicability and the calculation of derivatives may be costly. Also, the discrete control approach chosen by Macian et al. causes difficulties and slows down the optimization. However, the DM with NLP overall presents strongly improved results and performance over DP and PMP. Based on their approach, additions to the model have been implemented to improve the results and analyze the applicability with respect to battery powered traction.

\section{Methodology - BEMU Model}

The first and most fundamental change from the approach by Macian et al. is the replacement of discrete by continuous control to simplify the control problem. Moreover, a continuous controller is appropriate for electric traction, especially for future applications with automated driving. We limited the power control setting $N$ to a range between -200 and $+100 \%$. Here $+100 \%$ denotes full tractive effort, 0 the idle position, $-100 \%$ full regenerative braking and $-200 \%$ full regenerative braking with additional full power of the mechanic brakes.

$$
N_{i} \in[-200,100] \quad \forall i
$$

With this change the optimization process is reduced to one compared to the two iterations conducted by Macian et al. in order to handle the discrete variables.

At this point, the fundamental assumption for the function type between collocation points had to be made. Our approach for the base state and actuator variables was to assume that the power control setting is approximated by a piecewise constant and the velocity $v$ by a piecewise linear function. This is in agreement with the assumptions made by Macian et al. and other research. ${ }^{8,13}$ With this being the foundation of all following models in this work, the approximation error (i.e. the difference between reality and modeled quantities) that is introduced here has to be kept in mind. However, this error can be limited by intelligent grid setup as shown later on.

In order to implement a BEMU model, a novel electric drivetrain and a battery model had to be developed. In this model, recuperation of brake energy was included via generators at the traction units as well as two different energy sources and sinks (overhead catenary and 
This is a manuscript, which has been accepted for publication.

Published in: Proceedings of the Institution of Mechanical Engineers, Part F: Journal of Rail and Rapid Transit. October 2020.

battery). A schematic overview of the important components is given in Fig. 1. Additionally, auxiliary loads such as heating, ventilation, lights and cooling were considered. ${ }^{15}$ All components are connected to the core DC-link on the train and for the energy throughputs load-dependent efficiencies and idle losses were integrated.

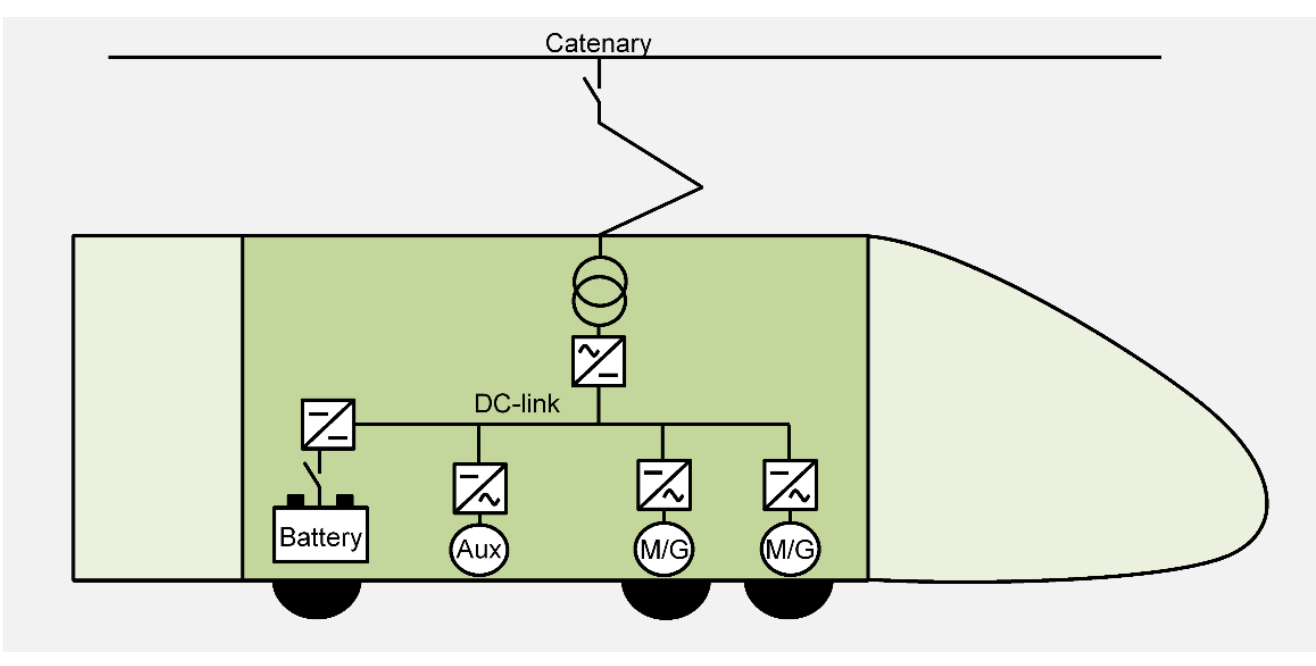

Figure 1. Schematic representation of the train model with all electric components.

\section{Differential Equations and Constraints}

The equation of motion defines the relation between acceleration $\dot{v}$ and traction or braking forces $F_{\text {trac/brake }}$ and resistance force $F_{\text {res }}$, divided by the mass $m$ (static and rotational):

$$
\dot{v}=f(N, v)=\frac{F_{\text {trac } / \text { brake }}(N, v)-F_{\text {res }}(v)}{m_{\text {static }}+m_{\text {rot }}}
$$

It was solved, as done before, with a modified Euler method, which is called the implicit midpoint rule. The left hand side of the differential equation is approximated by a difference quotient

$$
\dot{v} \approx \frac{v_{i+1}-v_{i}}{\Delta t}
$$

The travel time between two grid points $\Delta t_{i}$ is determined by the distance $\Delta s_{i}=s_{i+1}-s_{i}$ and the mean velocity $\bar{v}_{i}$ in that segment:

$$
\Delta t_{i}=\frac{\Delta s_{i}}{\bar{v}_{i}}
$$

The mean velocity is described as the average between the neighboring grid points, because the velocity is assumed to be linear between points.

$$
\bar{v}_{i}=\frac{v_{i}+v_{i+1}}{2}
$$

The right hand side is evaluated between collocation points. With the assumptions of piecewise constant control and linear velocity this results in

$$
\dot{v}_{i}=f\left(N_{i}, \bar{v}_{i}\right)
$$


This is a manuscript, which has been accepted for publication.

Published in: Proceedings of the Institution of Mechanical Engineers, Part F: Journal of Rail and Rapid Transit. October 2020.

Publisher Version:10.1177/0954409720970002

The forces for traction and braking are given by the quotient of the power at wheels $P_{\mathrm{w}}$ and $\bar{v}_{i}$ :

$$
F_{\text {trac } / \text { brake }}(N, v)=\frac{P_{\mathrm{w}}\left(N_{i}\right)}{\bar{v}_{i}}
$$

Resistance forces are approximated by the Davis formula (with coefficients $\tilde{A}, \tilde{B}$ and $\tilde{C}$ ), ${ }^{16}$ also considering gradients and curve resistances with Röckl's formula (parameters $D_{1}$ and $D_{2}$, gravitational constant $g),{ }^{17}$ similar to Macian et al. ${ }^{13}$

$$
F_{\text {res }}(v)=\tilde{A}+\tilde{B} \bar{v}_{i}+\tilde{C} \bar{v}_{i}^{2}+m g\left(\sin \left(\arctan \left(\tilde{\alpha}_{i}\right)\right)+\frac{D_{1}}{r_{i}-D_{2}}\right)
$$

Gradients $\tilde{\alpha}_{i}$ and radii $r_{i}$ are given as piecewise constant inputs. However, curve resistances were disregarded for the simulations in this work because of insufficient data.

Additionally to the equation of motion, multiple other constraints were adopted from Macian et al. with slight changes:

- The travel time for each section $j$ between stops has to be shorter or equal to the given timetable $\left(t_{\mathrm{tab}, j}\right)$. These integrals can be approximated by a sum with the assumptions from equation (4) and (5):

$$
\int_{t_{\text {start }}}^{t_{\text {end }}} \mathrm{d} t \approx \sum_{i} \frac{\Delta s_{i}}{\bar{v}_{i}} \leq t_{\mathrm{tab}, j} \forall i \text { in one section } j
$$

- The maximum positive force at wheels $F_{\text {trac }}$ is limited by the adhesion limit $F_{\text {adh }}$.

$$
F_{\text {trac }, i} \leq F_{\text {adh }} \forall i
$$

- The same restriction was applied for the electric brake force $F_{\text {brake }}$ with the force limit of the generator $F_{\text {gen }}$, which has a negative value.

$$
F_{\text {brake }, i} \geq F_{\text {gen }} \forall i
$$

Furthermore, we added a comfort constraint on the de- and acceleration $a$ :

$$
a_{\text {brake,max }} \leq a_{i} \leq a_{\text {trac,max }} \forall i
$$

The acceleration in each grid step is constant due to linear velocity and can be calculated by the change in velocity in one step. With assumptions in equation (4) and (5), this yields:

$$
a_{i}=\frac{v_{i+1}-v_{i}}{\Delta t_{i}}=\frac{v_{i+1}^{2}-v_{i+1}^{2}}{2 \Delta s_{i}}
$$

\section{ESS Model and Constraints}

Additional constraints were required to properly describe the behavior of the ESS system. The implementation accounts for a battery system with a variable number of battery branches in a parallel circuit. ${ }^{18}$ One branch was modeled as a power source with power losses in an internal resistance. This is illustrated in Fig. 2. Here, the main parameters of interest are open circuit voltage $U_{\mathrm{OC}}$, nominal capacity $C_{\mathrm{nom}}$, internal resistance $R_{\text {int }}$ and the number of branches $n_{\text {branch. }}$. All of these variables were kept constant for simplicity except for $U_{\mathrm{OC}}$ which was modeled as dependent on the State of Charge (SoC). With this simplified model, it is easy to feed the algorithm with publicly available battery data. Besides this, it also has the potential to provide significantly faster computational times then more complex approaches while still yielding very similar results. Following the proposed model, two new solution variables were 
This is a manuscript, which has been accepted for publication.

Published in: Proceedings of the Institution of Mechanical Engineers, Part F: Journal of Rail and Rapid Transit. October 2020.

Publisher Version:10.1177/0954409720970002

introduced to describe the state and control of the additional system: SoC and the current $I$. It is assumed that both new variables are piecewise constant.

The current $I$ is given in A, the SoC in \%. $I$ is limited by a maximum charge and discharge current for the battery, bounds for $S o C$ can be set as an operational window for the battery.

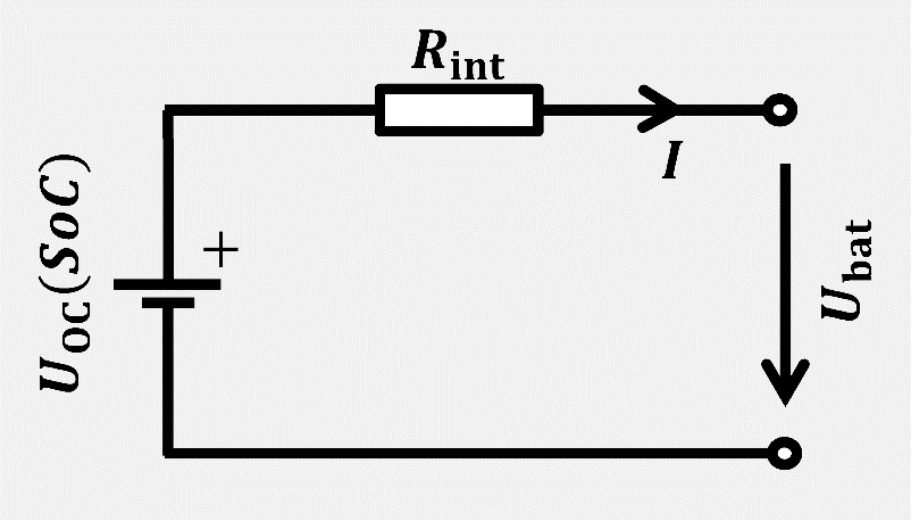

Figure 2. Schematic representation of the train model with all electric components.

To obtain $I$ in each step, a balance around the battery can be formulated:

$$
\begin{array}{r}
P_{\text {batt }, \mathrm{DC}}=n_{\text {branch }}\left(U_{\mathrm{bat}} I_{\mathrm{branch}}\right) \\
=n_{\text {branch }}\left(\left(U_{\mathrm{OC}}-R_{\text {int }} I_{\text {branch }}\right) I_{\text {branch }}\right)
\end{array}
$$

Here, $P_{\text {batt,DC }}$ depicts the battery power which matches the power from the DC-link (sum of motor/generator and charging from catenary) including losses in the battery converter. $n_{\text {branch }}$ is the number of parallel battery branches, $U_{\text {bat }}$ the overall battery voltage. To obtain $I$, the quadratic equation has to be solved. Only one of the two solutions is physically valid:

$$
I=n_{\text {branch }}\left(\frac{U_{\mathrm{OC}}}{2 R_{\mathrm{int}}}-\sqrt{\left(\frac{U_{\mathrm{OC}}}{2 R_{\mathrm{int}}}\right)^{2}-\frac{P_{\mathrm{batt}, \mathrm{DC}}}{R_{\mathrm{int}} n_{\mathrm{branch}}}}\right)
$$

Besides that, a system equation for the battery to model $S o C$ is needed. In this simple internal resistance model, $S o C$ is given by (cf. Waheed ${ }^{19}$ ):

$$
\text { SoC }=S o C_{\text {start }}-\int_{0}^{t_{\text {end }}} \frac{I}{C_{\text {nom }}} \mathrm{d} t \cdot 100 \%
$$

In the discretized model, the integral is, again, approximated by a sum over piecewise constant currents. Between two grid points this yields:

$$
S_{o} C_{i+1}=S o C_{i}-\frac{I_{i}}{C_{\mathrm{nom}}} \Delta t_{i} \cdot 100 \%
$$

For the operation in both catenary and catenary-free sections on the same track, it is imperative to determine a proper battery control strategy. This optimization of the charging and discharging control is conducted alongside the train control optimization. This way, it is made sure that the power control is adapted to battery operation in catenary-free segments, the battery is efficiently recharged under catenary and, most importantly, boundary 
This is a manuscript, which has been accepted for publication.

Published in: Proceedings of the Institution of Mechanical Engineers, Part F: Journal of Rail and Rapid Transit. October 2020.

Publisher Version:10.1177/0954409720970002

conditions can be given for the $S o C$. With imposing a balanced $S o C$ at start and end of the route, comparability between different scenarios and setups can be guaranteed.

For charge control two additional variables are defined:

- A blend factor $\theta$, given in \%, to determine whether the power is taken from or fed into battery (100\%) or catenary (0\%). In catenary-free operation, $\theta$ is fixed to $100 \%$.

- A second blend factor $\epsilon$, given in \%, to define the power with which the battery is recharged under catenary. $\epsilon=100 \%$ implies a charging power of $P_{\text {max,bat }}$ with the specified highest charging rate.

\section{Objective function}

The general objective function is defined as an integral of a cost function over the whole domain. This can, again be approximated by a sum with equations (4) and (5).

$$
f(x)=\int_{t_{\text {start }}}^{t_{\text {end }}} \operatorname{Cos} t \mathrm{~d} t \approx \sum_{i=1}^{n-1} \operatorname{Cost}_{i} \frac{\Delta s_{i}}{\bar{v}_{i}}
$$

In this work, the objective was to minimize electric energy consumption. Because the final SoC is given in form of a boundary condition, it is possible to optimize energy consumption at the catenary only. The amount of energy consumed by the battery is optimized indirectly via charging power and the two blend factors, which are linked by the battery current constraint. With $P_{\text {trac,cat }}$ as tractive power at the catenary and $P_{\text {ch,cat }}$ as the additional charging power at catenary, the cost function for catenary operation results to:

$$
\operatorname{Cost}(N, \theta, \epsilon)=(1-\theta) P_{\text {trac,cat }}(N)+\epsilon P_{\text {ch,cat }}
$$

At this state of the implementation, it is important to point out that adding traction and charging power with $\epsilon$ introduces a model inaccuracy because efficiencies and idle losses are calculated preemptively as a function of power control setting. However, the actual power throughput through some of the components such as the transformer differs since the power from battery to catenary and vice versa is calculated only during the optimization. For further improvement, efficiency functions with multiple dependencies $\eta(N, \theta, \epsilon)$ could be introduced. This would, however, lead to increased computational times and, hence, was avoided in this first approach. The individual error introduced by this approximation might be estimated by a backwards simulation of the results.

\section{Further Adaptions}

In addition to the modifications described above further measures were introduced for a more accurate and faster optimization.

Firstly, as velocity appears in the denominator in the traction force calculation of the force constraints and the equation of motion, a minimum numerical speed $v_{\min }$ was introduced to ensure a robust solution process. Together with the speed limits this yields the computational bounds for velocity:

$$
v_{i} \in\left[v_{\min }, v_{\max , i}\right] \quad \forall i
$$

Since auxiliary loads were included in the model, these have to be covered during station stops as well. For simplicity they are assumed constant in our approach. However, together with the 
This is a manuscript, which has been accepted for publication.

Published in: Proceedings of the Institution of Mechanical Engineers, Part F: Journal of Rail and Rapid Transit. October 2020.

Publisher Version:10.1177/0954409720970002

new requirements of reaching the final $S o C$ with proper charging control, this causes a need for optimizing the whole route in one process, including a handling of stops. The stops are modeled as small, virtual grid steps that correspond to the time it takes to drive this step size at $v_{\text {min }}$.

Macian et al. realized twice continuously differentiable functions, which are necessary for the NLP solver, by utilizing Fourier series and Gaussian functions. ${ }^{13}$ Since our method includes a lot of additional input functions, a more general approach was used. With a formulation by Jimenez-Fernandez et al., piecewise linear functions can be transformed by smoothing the salient, non-differentiable points. ${ }^{20}$ This method is illustrated in Fig. 3, the mathematical formulation is:

$$
f(x)=\hat{A}+\hat{B} x+\sum_{i=1}^{n_{\text {salient }}} \hat{C}_{i} \ln \left(1+\mathrm{e}^{-\alpha\left(x-\widehat{\beta}_{i}\right)}\right)
$$

Here, $\hat{A}, \hat{B}, \hat{C}_{i}$ and $\hat{\beta}_{i}$ denote parameters that are calculated from the known points; $n_{\text {salient }}$ is the number of breakpoints between piecewise linear sections and $\alpha$ is a smoothing parameter. With increasing $\alpha$ the result gets closer to the piecewise linear function. However, if $\alpha$ is too big, the function might not be defined in the whole domain. To achieve the best possible result for each individual smoothing process, the highest possible $\alpha$ is determined. Typical values for our input functions are between 2 and 4 .

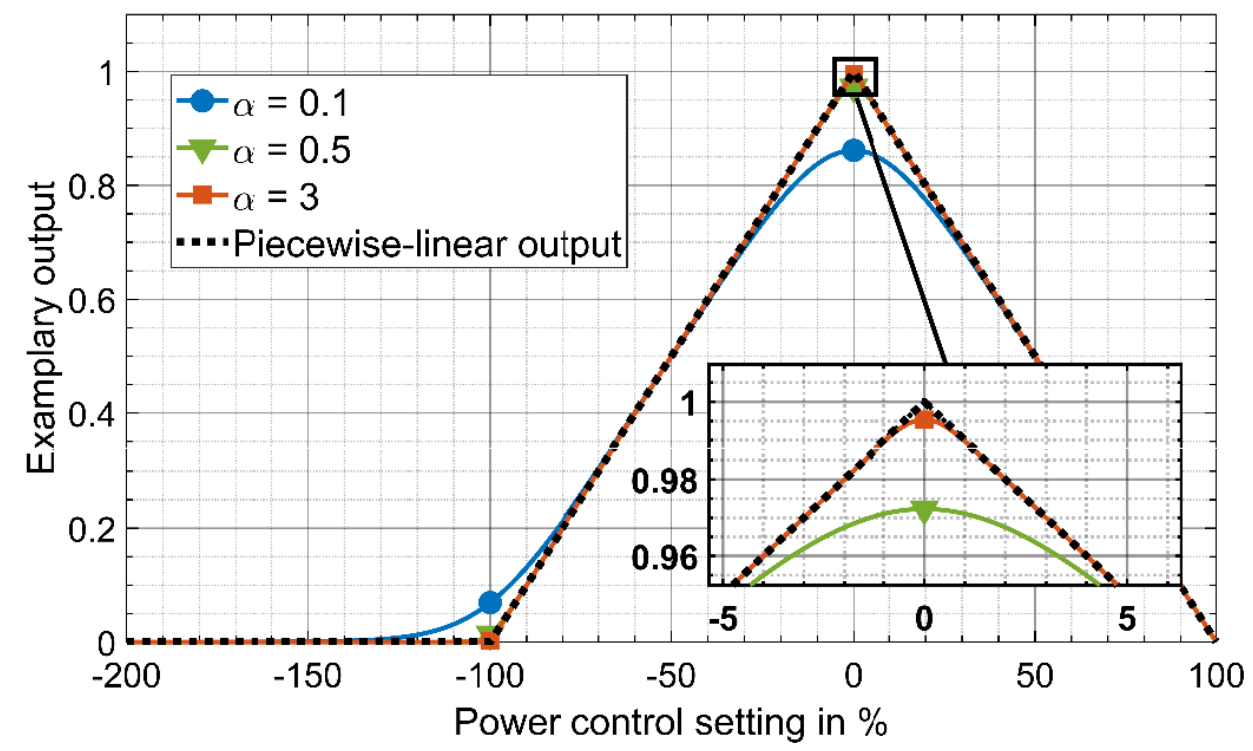

Figure 3. Exemplary piecewise linear function versus power control setting and the associated smoothed function with different.

The last adaption was to include second derivatives. Instead of using numerical approximation of second derivatives by a Quasi-Newton method ${ }^{21}$ the information about second derivatives for both objective function and all constraints is passed explicitly in a single matrix, which is called the Hessian $H$ of the Lagrangian. 
This is a manuscript, which has been accepted for publication.

Published in: Proceedings of the Institution of Mechanical Engineers, Part F: Journal of Rail and Rapid Transit. October 2020.

Publisher Version:10.1177/0954409720970002

\section{Numerical Parameter Study and Grid Calibration}

To develop a fast and robust algorithm with little computational requirements, the numerical setup was analyzed and calibrated. In particular, these are the grid setup and the initial solution.

The most impactful parameters are the amount and positioning of the collocation/grid points. The grid setup always implies a trade-off between computational speed and accuracy. The more grid points are used, the smaller the approximation error due to assumptions of piecewise function made in the Methodology become. On the other hand, the computation effort increases usually more than linearly with an increasing number of grid points. A grid study was conducted on a short, synthetic test route that is illustrated in Fig. 4 . With this route, multiple series of tests including very high resolutions could be conducted. As a test vehicle, a generic regional BEMU was designed with realistic parameters from state-of-the-art BEMU prototypes and other regional trains. ${ }^{22-26}$ The key vehicle characteristics are given in Table 1. For the simulations, a train occupancy rate of $80 \%$ of the total passenger capacity was assumed, with $75 \mathrm{~kg}$ per passenger. The rotational mass $m_{\text {rot }}$ was set to $5 \%$ of $m_{\text {static }}$.

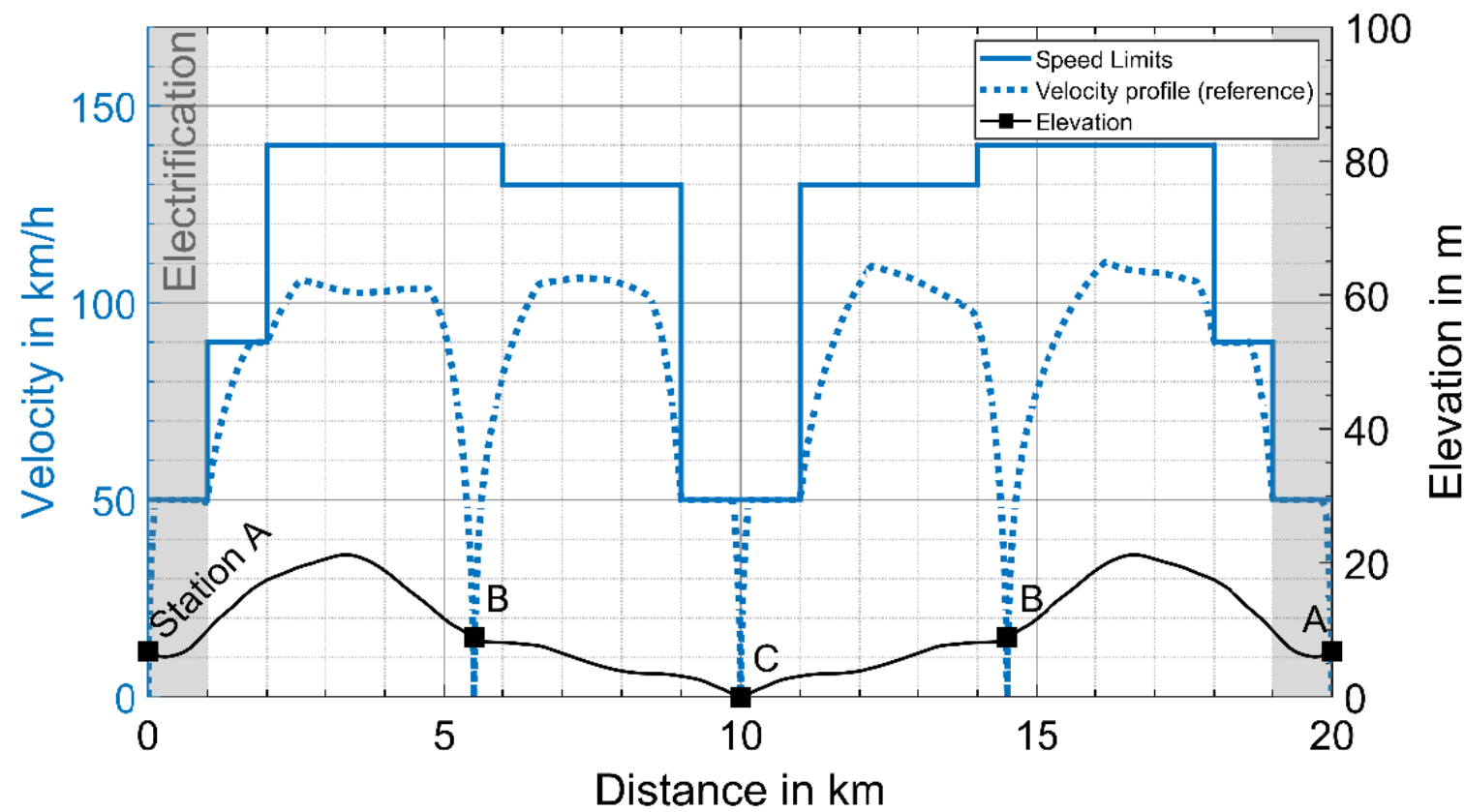

Figure 4. Track characteristics of the test track with elevation, speed limits, stops and the reference velocity profile.

As measured data was not available, the reference for the grid study has been derived by increasing the number of grid points until the results converge to a solution that is not changed significantly by a further increase of resolution. In Fig. 4, the corresponding velocity profile for the reference case is depicted.

With the use of refinement layers around critical points, the resolution in the rest of the domain can be lower without a big loss of accuracy. This means that areas with rather constant solution behavior such as coasting phases can be mapped with bigger grid steps than strong de- and acceleration phases. After various test rounds, it was found that a refinement layer around stations combined with an equidistant grid in the rest of the route yield stable results. 
This is a manuscript, which has been accepted for publication.

Published in: Proceedings of the Institution of Mechanical Engineers, Part F: Journal of Rail and Rapid Transit. October 2020.

The refinement layer was set up with a first grid step of $10^{-3} \mathrm{~m}$ before and after the stop. From this smallest step the following steps grow in size with a widening factor of 1.5 until the equidistant base step size is met.

Table 1. Key characteristics of the generic BEMU.

\begin{tabular}{ll}
\hline Characteristic & Value \\
\hline Length & $55 \mathrm{~m}$ \\
Mass $\boldsymbol{m}_{\text {static }}$ (empty) & $120 \mathrm{t}$ \\
Passenger Seats & 150 \\
Maximum Velocity & $140 \mathrm{~km} / \mathrm{h}$ \\
Max. De-/Acceleration & $1 \mathrm{~m} / \mathrm{s}^{2}$ \\
Max. Power at Wheels & $2 \mathrm{MW}$ (catenary), 1 MW (battery) \\
Davis Coefficients $\widetilde{\boldsymbol{A}}, \widetilde{\boldsymbol{B}}, \widetilde{\boldsymbol{C}}$ & $1956 \mathrm{~N}, 17.6 \frac{\mathrm{N}}{\mathrm{m} / \mathrm{s}^{\prime}}, 2.59 \frac{\mathrm{N}}{(\mathrm{m} / \mathrm{s})^{2}}$ \\
Motor/Generator Limit $\boldsymbol{F}_{\text {adh }}, \boldsymbol{F}_{\text {gen }}$ & $109956 \mathrm{~N}$ \\
Nominal Battery Capacity & $616 \mathrm{Ah}$ \\
Nominal Open Circuit Voltage $\boldsymbol{U}_{\mathbf{O C}}$ & $790 \mathrm{~V}$ \\
Internal Resistance $\boldsymbol{R}_{\text {int }}$ & $0.65 \Omega$ \\
\hline
\end{tabular}

In Fig. 5, the simulation test results for various base step sizes are shown. The graph shows deviations in the results as well as the computational time for an optimization run with the simplest initial guess i.e. equal values for the solution variables in the whole domain. The deviations in energy and velocity are calculated against the reference (with a resolution of 0.5 m-steps).

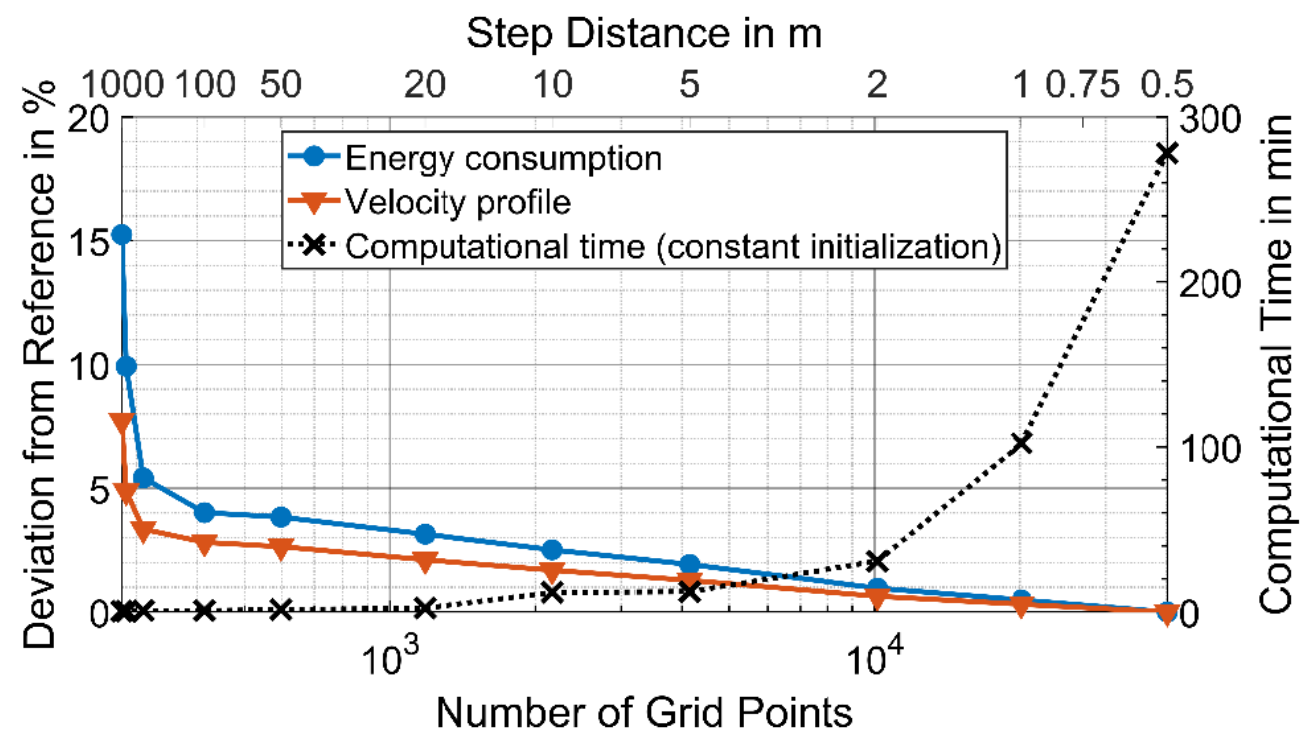

Figure 5. Track characteristics of the test track with elevation, speed limits, stops and the reference velocity profile. 
This is a manuscript, which has been accepted for publication.

Published in: Proceedings of the Institution of Mechanical Engineers, Part F: Journal of Rail and Rapid Transit. October 2020.

Publisher Version:10.1177/0954409720970002

The numerical analysis showed that the discretization error for all grids with base steps smaller than $100 \mathrm{~m}$ steps is limited to $5 \%$. The grid with $100 \mathrm{~m}$-steps was used for further optimization because of the most advantageous trade-off between time and accuracy. The computational time (Intel ${ }^{\circledR}$ Core $^{\mathrm{TM}}$ i7-8665U CPU@1.9 GHz and 16 GB RAM) is decreased from the magnitude of hours ( $4 \mathrm{~h} 38 \mathrm{~min}$ for $0.5 \mathrm{~m}$-steps) to $49.6 \mathrm{~s}$ while still yielding accurate results. Since the energy consumption has a positive deviation compared to the reference in all cases, there is also no danger of overestimating the energy saving potential. Only one other analysis of grid calibration was found in railway trajectory optimization literature, conducted by Wu et al. with a PMP optimization. ${ }^{8}$ They suggested an optimal step size of $100 \mathrm{~m}$, which matches with our results.

After calibrating the grid setup, the initial solution was analyzed as a second parameter. Tests of different approaches proved a strong influence on computational time, while the results concerning energy consumption and velocity did not vary considerably $(<1 \%)$. The fastest overall performance was achieved with a trajectory from a preceding optimization with IPOPT that respects all constraints, but is not optimized $(f=0)$ and does not consider the battery model. The initial battery variables were theoretically estimated using the system equations introduced above.

This approach further reduced the computational time with $100 \mathrm{~m}$ base steps from $49.6 \mathrm{~s}$ to $4.9 \mathrm{~s}(0.8 \mathrm{~s}$ for the initial optimization and $4.1 \mathrm{~s}$ for the final run) by approximately $90 \%$, hence demonstrating that an intelligent initial guess is crucial.

The procedure introduced above can contribute to design accurate and fast numerical algorithms. With this calibration, our algorithm yields fast results while still being within a high numerical accuracy. Overall the computational time compared to the reference was reduced from the magnitude of hours to only $4.9 \mathrm{~s}$ by utilizing appropriate refinement layers in combination with coarse base grid steps $(100 \mathrm{~m})$ and an intelligent initialization. Despite the significant reduction of grid points, the results were still within a $5 \%$ numerical error margin.

\section{Use Case (Results)}

With the optimized setup, the algorithm was applied to the use case of a regional line in Germany from Hagen to Warburg with a length of $152 \mathrm{~km}$. A geographical overview of the route is given in Fig. 6 .

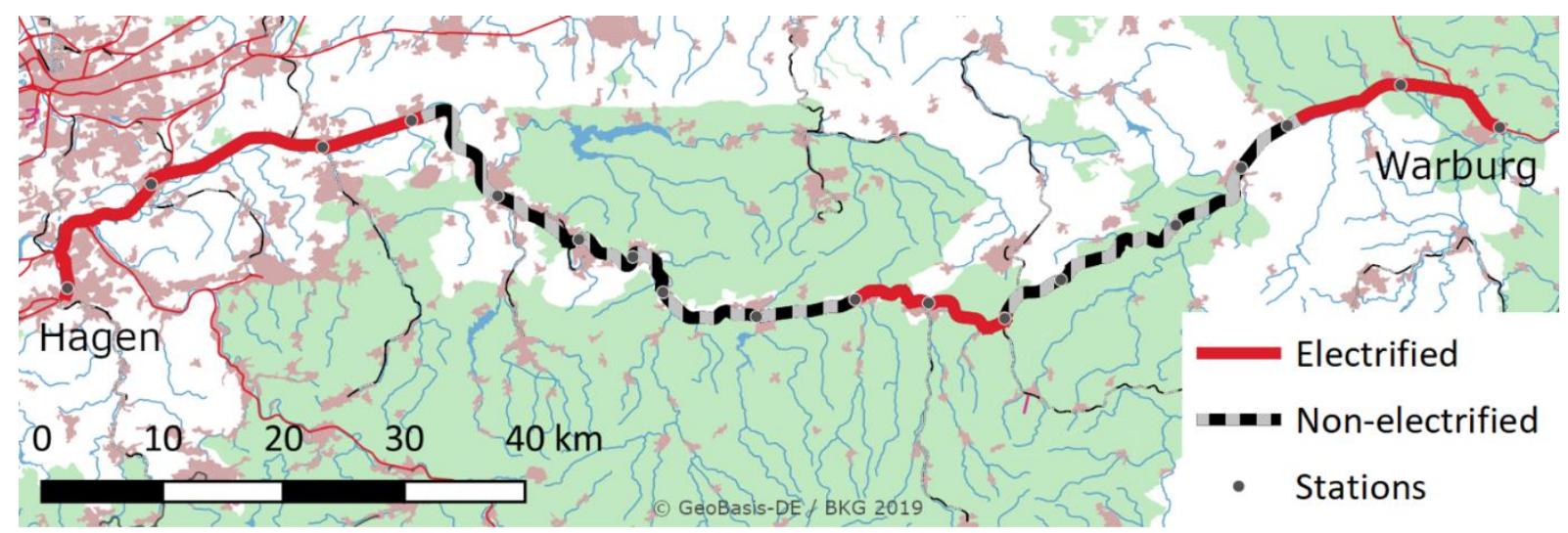

Figure 6. Map of the route Hagen-Warburg with stations and assumed electrification (illustration by DLR). 
This is a manuscript, which has been accepted for publication.

Published in: Proceedings of the Institution of Mechanical Engineers, Part F: Journal of Rail and Rapid Transit. October 2020.

Publisher Version:10.1177/0954409720970002

Line speed limits, station stops and electrified sections are shown in Fig. 7. This line is equipped with overhead lines in the beginning $(14.1 \mathrm{~km})$ and the end $(2.5 \mathrm{~km})$ of the track. In our analysis, the electrification was extended by $16 \mathrm{~km}$ on each side and a catenary island (14.2 $\mathrm{km}$ ) in the middle of the track to yield a partly electrified scenario that is feasible with the generic BEMU introduced above.

Simulations with the proposed algorithm and abovementioned calibrated parameters were carried out in order optimize the operation for this use case. Here, the optimization converged without constraint violations in approximately $12 \mathrm{~min}(0.4 \mathrm{~min}$ initialization and $11.6 \mathrm{~min}$ optimization) and yielded a total energy consumption at catenary of $1.1 \mathrm{MWh}$. The optimized solution was compared to a simulation without minimization of the energy that follows the same constraints and boundary conditions (including the timetable). Here, the optimization yields an improvement of $8.3 \%$ in energy consumption at catenary, which clearly demonstrates the energy saving potential of the algorithm. The optimized net energy at battery was $528 \mathrm{kWh}$, including $-256 \mathrm{kWh}$ of recuperated energy, which corresponds to a recuperation factor of $23.3 \%$. This factor was calculated as recuperated energy at battery divided by energy consumption at catenary. The non-optimized simulation only yields a recuperation factor of $16.8 \%$, while at the same time amplifying battery stress due to increased energy output at battery. The key simulation results are summarized in Table 2 . Additionally, the energy costs for a single roundtrip were calculated (see Table 2). For this economic evaluation, electricity prices at catenary within the German network by Deutsche Bahn were used. In 2019, buying electricity was charged with 5.69 cents/kWh, while feeding recuperated energy into the grid gave a revenue of 2.73 cents/kWh. ${ }^{27}$ Assuming a line schedule with four trains going three times per day (4380 roundtrips per year based on timetables by Deutsche Bahn ${ }^{28}$ ), this leads to savings of around $31100 €$ per year only due the reduced energy consumption when compared to the non-optimized simulation. It has to be taken into account that these results depend heavily on the chosen line and vehicle. 
This is a manuscript, which has been accepted for publication.

Published in: Proceedings of the Institution of Mechanical Engineers, Part F: Journal of Rail and Rapid Transit. October 2020.

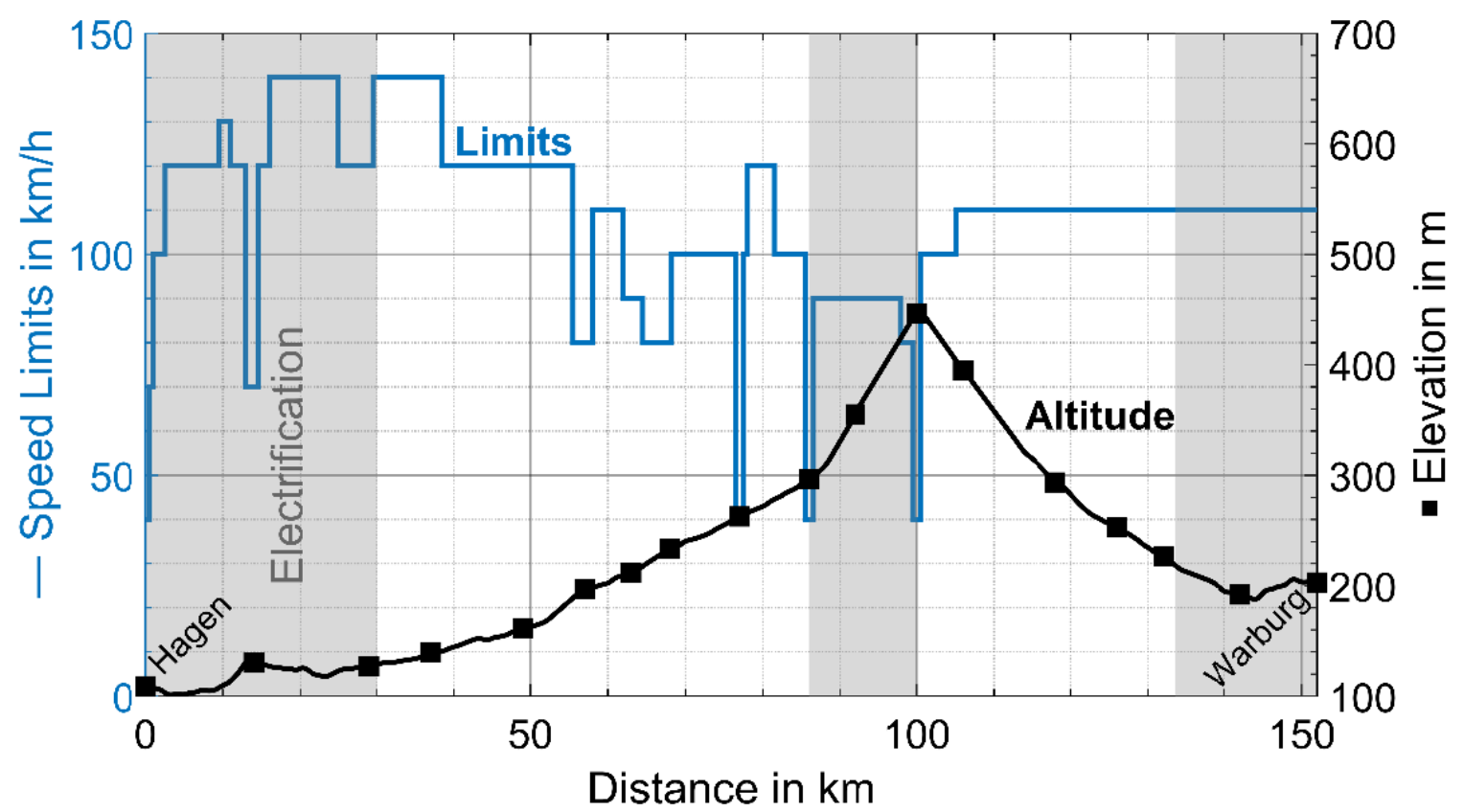

Figure 7. Track characteristics of the route Hagen-Warburg with elevation, speed limits, stops and assumed electrification.

Table 2. Key simulation results for the regional use case Hagen - Warburg (Germany).

\begin{tabular}{lrr}
\hline Results per roundtrip & $\begin{array}{r}\text { Non-optimized } \\
\text { simulation }\end{array}$ & $\begin{array}{r}\text { Optimized } \\
\text { operation }\end{array}$ \\
\hline Computational time & $1 \mathrm{~min}$ & $12 \mathrm{~min}$ \\
Energy at catenary & $1.2 \mathrm{MWh}$ & $1.1 \mathrm{MWh}$ \\
Net energy at battery without catenary recharging & $590 \mathrm{kWh}$ & $528 \mathrm{kWh}$ \\
Recuperated energy at battery & $-201 \mathrm{kWh}$ & $-256 \mathrm{kWh}$ \\
Energy costs at catenary & $69.7 €$ & $62.6 €$ \\
\hline
\end{tabular}

The detailed optimized trajectory of the roundtrip simulation for this use case is depicted in Fig. 8. Here, subfigures 8 a) and e) show the whole trajectory, while subfigures b)-d) depict a representative segment of the route including five driving sections, for which the effects are discussed in detail:

The results in terms of applied traction power clearly adapt to the available power sources in the respective line section. For section (V), the typical phases of energy efficient driving (optimum-efficiency-acceleration, coasting and braking) can be seen (cf. Fig. 8 b). The nonelectrified sections (I)-(III) deviate from this behavior. This is due to the limited power of the battery, which results in a maximum control setting of approximately $50 \%$ as seen in the graph (cf. Fig. 8 c-d). The inhibited acceleration capacity leads to longer acceleration phases with shorter or non-existent coasting between stations. In section (IV), it can be seen that the train accelerates again, once it enters the electrified part and makes use of the catenary supply and the higher efficiency power setting. 
This is a manuscript, which has been accepted for publication.

Published in: Proceedings of the Institution of Mechanical Engineers, Part F: Journal of Rail and Rapid Transit. October 2020.

Publisher Version:10.1177/0954409720970002

Concerning our goal to increase the level of detail of the modeled train, some differences to the results by Macian et al. can be found. Firstly, a more realistic braking behavior was achieved. Our results describe a smooth transition from coasting to regenerative breaking (cf. Fig. $8 \mathrm{a}-\mathrm{c}$ ) due to the additional comfort constraint for deceleration and due to recuperation, which makes early and slow braking more profitable than using the mechanical brakes and dissipating the kinetic energy.

Additionally, the effects of load-dependent efficiencies on power control settings are visible. In particular, idle operation is mostly avoided and the coasting phase is rather a slow transition from tractive effort to regenerative braking than a simple zero-position control (cf. Fig. $8 \mathrm{c}$ ). This is due to the bad efficiency of idle operation with losses in the electric components. On the other hand acceleration is never carried out with full power effort, but rather around a maximum of $60 \%$, which is the most efficient power control setting with these specific efficiencies.

In Fig. $8 \mathrm{~d}$, the optimized power at catenary for the chosen segment can be seen. One part of this power is used to recharge the battery while driving (dotted line). By minimizing this part, the power at battery is implicitly optimized in the non-electrified sections and there shows a similar behavior as the tractive power at catenary. As expected, the regenerative energy during braking is not fed back into the catenary but utilized to recharge the battery since feeding back and then recharging the battery from catenary has a lower overall efficiency. Similarly, the tractive power in electrified sections is always supplied by catenary, since using the battery would imply additional losses. In Fig. 8e, the resulting $\mathrm{SoC}$ is depicted. On electrified sections, the battery is recharged in order to fulfil the requirement of a balanced state of charge in the beginning and the end.

The use case results clearly proved the applicability of the algorithm on a partly electrified BEMU scenario. The solver converged to realistic results without any constraint violations. It was seen that both velocity profile and control adapt to the varying availability of power supply by catenary. As expected, regenerative energy is always fed into the battery if possible and tractive energy is preferably supplied by catenary if available. Therefore, this optimization leads to the maximum utilization of catenary and sparing use of battery. 
This is a manuscript, which has been accepted for publication.

Published in: Proceedings of the Institution of Mechanical Engineers, Part F: Journal of Rail and Rapid Transit. October 2020.

Publisher Version:10.1177/0954409720970002
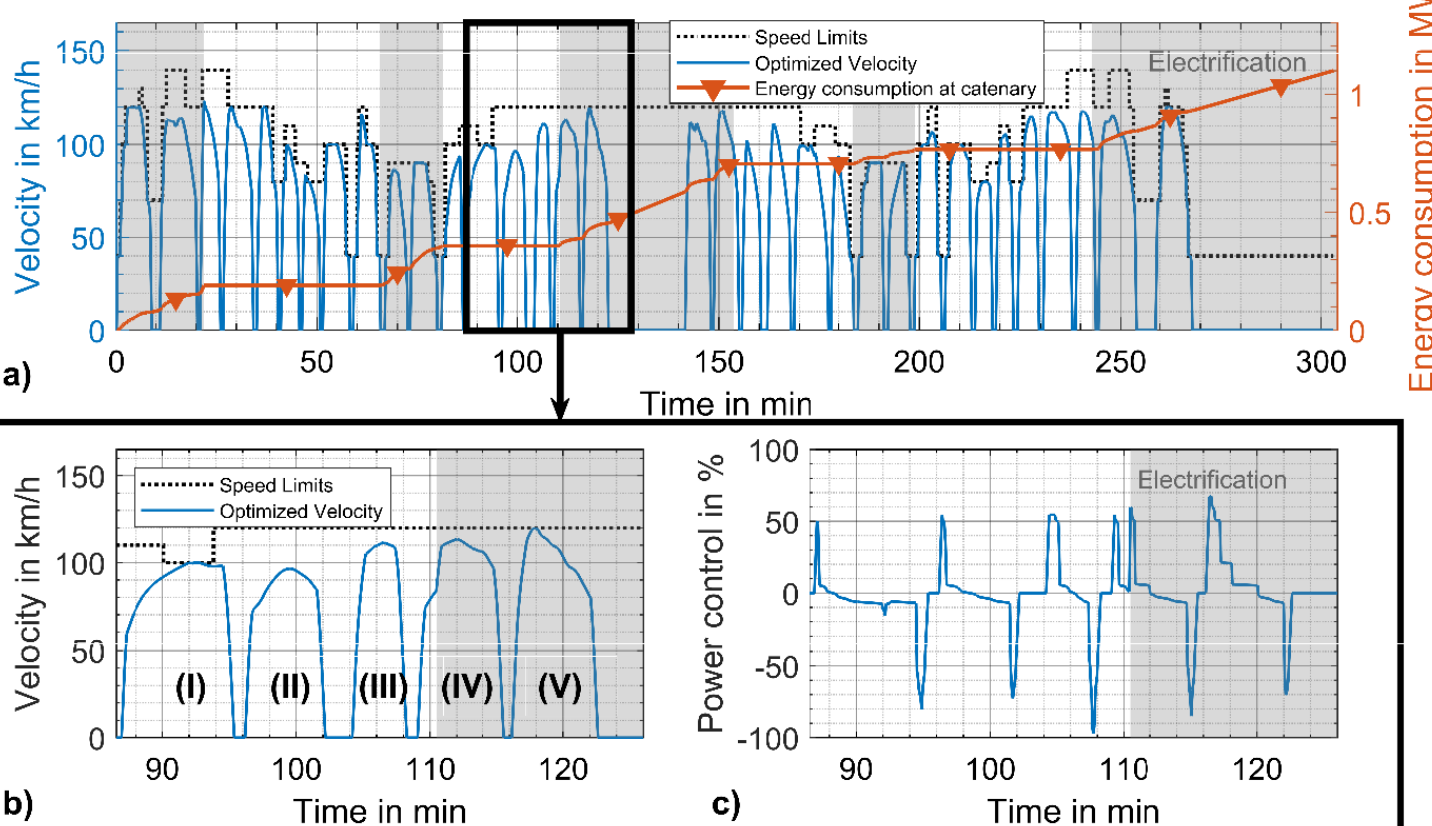

c) Time in min
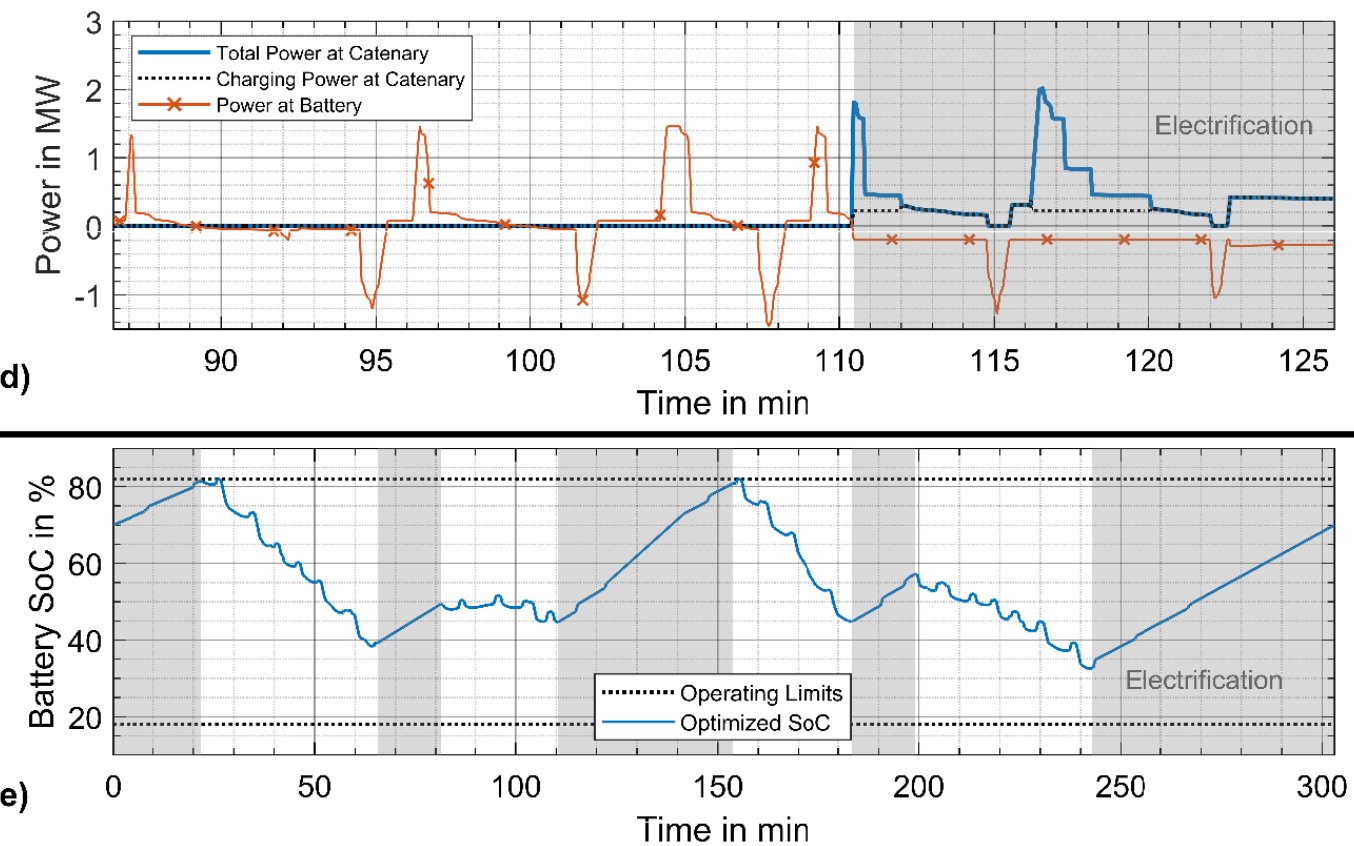

Figure 8. (a) Velocity profile and energy consumption at catenary for the whole round trip with turnover stopping time; (b) velocity profile for a representative segment including five driving sections; (c) power control setting for the segment; (d) power at catenary (total and for charging) and battery for the segment; e) resulting SoC for the whole round trip. 
This is a manuscript, which has been accepted for publication.

Published in: Proceedings of the Institution of Mechanical Engineers, Part F: Journal of Rail and Rapid Transit. October 2020.

Publisher Version:10.1177/0954409720970002

\section{Conclusions}

Our results proved that the DM optimization approach introduced by Macian et al. for diesel trains is also applicable to scenarios with battery electric railway vehicles and partly electrified tracks. To achieve this, the following modifications and extensions of the model were needed: a BEMU drivetrain model including an internal resistance battery model was implemented. Controls are now handled continuously and additional constraints were introduced. Furthermore, auxiliary loads and intermediate stops were considered in order to optimize the whole route at once. Utilizing a different approach to smooth non-differentiable functions and including second derivatives showed improved performance compared to the basic model by Macian et al. The numerical analysis yielded a suitable grid setup with a coarse base grid and refinement around stops. In combination with an intelligent initialization, a fast and at the same time accurate algorithm was designed.

The use case of a partly electrified route in Germany confirmed that our algorithm is able to handle the large optimization problem very well. Within $12 \mathrm{~min}$, we gained a converged minimum energy solution that showed the expected behavior of a BEMU, adapting to the changing conditions of the available power sources while also reducing battery degradation. Furthermore, the optimization algorithm prioritized the utilization of regenerative braking energy for battery charging over feeding electricity back in to the catenary as it is overall more energy-efficient. With an energy consumption of $1.1 \mathrm{MWh}$ at catenary, it was possible to recover $256 \mathrm{kWh}$ of regenerative braking energy in the presented use case. For this specific line, an energy recuperation factor of $23.3 \%$ was obtained. Compared to the non-optimized simulation, improvements of $8.3 \%$ in energy consumption at catenary could be achieved. Compared to manual operation, even higher improvements may be possible.

Besides the energy saving potentials shown by offline benchmark simulations, an online application of the algorithm is not ruled out, since it is able to take previous solution as an input to further speed up the optimization. Utilizing a pre-calculated optimal solution, our algorithm might be used to react to changing conditions during the journey and find an adapted optimal solution within an acceptable time.

Our algorithm presents a powerful tool to accelerate the change to a more sustainable transport sector as it can be used e.g. to plan and dimension the future operation of BEMUs. Some aspects of the work are still in development and need further improvements. The deficits of the current load-dependent efficiency-implementation could be overcome by a more complex approach. Further improvements could be the handling of dynamic auxiliary loads and a more detailed battery model including the allowance of short time peak currents. However, due to the high modularity of the algorithm, modifications like this and future additions such as hydrogen fuel cells as a power source are easily possible.

\section{Acknowledgements}

The authors would like to thank Athanasios Iraklis, who initially started the implementation of a direct method optimization algorithm during his time at DLR. 
This is a manuscript, which has been accepted for publication.

Published in: Proceedings of the Institution of Mechanical Engineers, Part F: Journal of Rail and

Rapid Transit. October 2020.

Publisher Version:10.1177/0954409720970002

\section{Declaration of Conflicting Interests}

The Authors declare that there is no conflict of interest.

\section{Funding}

This research was conducted within the DLR Next Generation Train project.

\section{References}

1. Pagenkopf J et al. Analysis of German diesel operated regional railway lines' patterns with regard to the application of battery and fuel cell electric trains. In: The Fourth International Conference on Railway Technology - Railways 2018, Sitges, Spain, 03-07 September 2018.

2. IEC 62864-1:2016. Railway applications - Rolling stock - Power supply with onboard energy storage system - Part 1: Series hybrid system.

3. Scheepmaker GM et al. Review of energy-efficient train control and timetabling. Eur J Oper Res 2017; 257: 355-376.

4. Miyatake M and Matsuda K. Energy Saving Speed and Charge / Discharge Control of a Railway Vehicle with On-board Energy Storage by Means of an Optimization Model. IEEJ Trans Electr Electron Eng 2009; 4: 771-778.

5. Noda $Y$ and Miyatake M. Methodology to apply dynamic programming to the energyefficient driving technique of lithium-ion battery trains. In: 2016 International Conference on Electrical Systems for Aircraft, Railway, Ship Propulsion and Road Vehicles \& International Transportation Electrification Conference (ESARS-ITEC), Tolouse, France, 02-04 November 2016.

6. Ghaviha $N$, Bohlin $M$ and Dahlquist E. Speed profile optimization of an electric train with on-board energy storage and continuous tractive effort. In: 2016 International Symposium on Power Electronics, Electrical Drives, Automation and Motion (SPEEDAM), Anacapri, Italy, 22-24 June 2016, pp. 639-644.

7. Mwambeleko JJ and Kulworawanichpong T. Battery electric multiple units to replace diesel commuter trains serving short and idle routes. J Storage Mater 2017; 11: 7-15.

8. Wu C et al. Train Speed Trajectory Optimization With On-Board Energy Storage Device. IEEE Trans Intell Transp Syst 2018; 20: 4092-4102.

9. Pröhl L et al. Baseline simulation results and assessment. Report for the OPEUS project (D3.2). 30 January 2019.

10. Schirmer T et al. Sub-Optimal Non-Linear Optimization of Trajectory Planning for the DLR Next Generation Train (NGT). In: The Fourth International Conference on Railway Technology - Railways 2018, Sitges, Spain, 03-07 September 2018.

11. Miyatake $\mathrm{M}$, Haga $\mathrm{H}$ and Suzuki S. Optimal speed control of a train with On-board energy storage for minimum energy consumption in catenary free operation. In: 13th European Conference on Power Electronics and Applications, Barcelona, Spain, 2009.

12. Xiao Z et al. Integrated Optimization of Speed Profiles and Power Split for a Tram with Hybrid Energy Storage Systems on a Signalized Route. In: Energies 2018; 11: 478. 
This is a manuscript, which has been accepted for publication.

Published in: Proceedings of the Institution of Mechanical Engineers, Part F: Journal of Rail and Rapid Transit. October 2020.

Publisher Version:10.1177/0954409720970002

13. Macian V et al. Application and benchmarking of a direct method to optimize the fuel consumption of a diesel electric locomotive. Proceedings of the Institution of Mechanical Engineers, Part F: Journal of Rail and Rapid Transit 2018; 232: 2272-2289.

14. Wächter A et al. GitHub Project: Ipopt https://github.com/coin-or/Ipopt (2020, accessed 04 June 2020).

15. Windemut I, Dittus $\mathrm{H}$ and Winter J. Einfluss der Bordnetzverbraucher auf die Reichweite von batterieelektrischen Triebzügen (BEMU) - Modellbasierte Analyse. In: Elektrische Fahrzeugantriebe und -ausrüstungen, Stuttgart, Germany, 12-13 September 2018.

16. Rochard BP and Schmid F. A review of methods to measure and calculate train resistances. Proceedings of the Institution of Mechanical Engineers, Part F: Journal of Rail and Rapid Transit 2000; 214: 185-199.

17. Ihme J. Schienenfahrzeugtechnik, Springer Fachmedien Wiesbaden, 2016, p. 38.

18. IEC 62928:2017. Railway applications - Rolling stock - Onboard lithium-ion traction batteries.

19. Waheed A. Modellierung, Echtzeitsimulation und genaue Prognose der Lebensdauer und Kosten einer LFP-Batterie am Beispiel eines A- und C-Segment E-Autos. PhD Thesis, Universität Duisburg-Essen, Germany, 2016.

20. Jimenez-Fernandez VM et al. Transforming the canonical piecewise-linear model into a smooth-piecewise representation. SpringerPlus 2016; 5: 1612.

21. Kawajir Y et al. Ipopt Documentation, https://coin-or.github.io/Ipopt/index.html (2020, accessed 04 June 2020).

22. Alstom SA. Coradia iLint - Triebzug mit Energie aus Brennstoffzellen. Elektrische Bahnen, 01 October 2018, pp. 350-353.

23. Ernst J et al. Energy Baseline. Report for the FINE1-project (D3.1), 13 March 2018.

24. Abellio $\mathrm{GmbH}$. Bombardier Talent $2 \mathrm{EMU}$, https://nl.abellio.de/nl/mitteldeutschland/unternehmennews/fahrzeugewerkstatt/bombardier-talent-2-emu (accessed 04 June 2020).

25. Von Mach $S$ et al. Die Entwicklung und Markteinführung des TALENT 3 Batterietriebzuges. Eisenbahntechnische Rundschau, 15 November 2018, pp. 115-119.

26. Schrader S. Stadler zeigt Flirt-3-Batteriezug. Schweizer Eisenbahn-Revue, 28 December 2018, p. 652.

27. DB Netze. Preisblatt für die Nutzung des $16,7-\mathrm{Hz}-$ Bahnstromnetzes (Bahnstromnetz) gültig ab 01.01.2019, https://www.dbenergie.de/dbenergiede/netzbetreiber/bahnstromnetz/veroeffentlichungen-netzzugang-bahnstrom (accessed 03 September 2020).

28. Deutsche Bahn AG. Elektronisches Kursbuch, http://kursbuch.bahn.de/hafas/kbview.exe (2020, accessed 03 September 2020). 